\title{
The walnut JrVHAG1 gene is involved in cadmium stress response through ABA-signal pathway and MYB transcription regulation
}

\author{
Zhenggang Xu ${ }^{1,2}, \mathrm{Yu} \mathrm{Ge}^{3}$, Wan Zhang ${ }^{1}$, Yunlin Zhao ${ }^{1 *}$ and Guiyan Yang ${ }^{4^{*}}$
}

\begin{abstract}
Background: Vacuolar $\mathrm{H}^{+}$-ATPase (V-ATPase) is a vital protein complex involved in abiotic stress response in plants. The G subunit of Juglans regia (JVVHAG1) was previously identified as a drought tolerance-related gene involved in the ABA (abscisic acid)-signal pathway. Heavy metal stress is becoming a major detriment for plant growth, development, and production. In order to understand the role of JVHAG1, the potential function mechanism of JVHAG1 exposed to $\mathrm{CdCl}_{2}$ stress was confirmed in this study.

Results: Transcription of JrVHAG1 was induced by ABA and increased to 58.89-fold (roots) and 7.38-fold (leaves) and by $\mathrm{CdCl}_{2}$ to 2.65- (roots) and 11.42-fold (leaves) relative to control, respectively. Moreover, when treated simultaneously with $\mathrm{ABA}$ and $\mathrm{CdCl}_{2}\left(\mathrm{ABA}+\mathrm{CdCl}_{2}\right)$, JrVHAG1 was up-regulated to 110.13- as well as 165.42 -fold relative to control in the roots and leaves, accordingly. Compared to the wild type (WT) Arabidopsis plants, the transgenic plants with overexpression of JrVHAG1 (G2, G6, and G9) exhibited increased seed germination rate, biomass accumulation, proline content, and activities of superoxide dismutase (SOD) and peroxidase (POD) under $A B A, C d C l_{2}$, and $\mathrm{ABA}+\mathrm{CdCl}_{2}$ treatments. In contrast, the reactive oxygen species (ROS) staining, malondialdehyde (MDA) content, hydrogen dioxide $\left(\mathrm{H}_{2} \mathrm{O}_{2}\right)$ content, as well as electrolyte leakage (EL) rates of transgenic seedlings were all lower than those of WT exposed to $\mathrm{ABA}, \mathrm{CdCl}_{2}$ and $\mathrm{ABA}+\mathrm{CdCl}_{2}$ stresses. Furthermore, a 1200 bp promoter fragment of JVVHAG1 was isolated by analyzing the genome of J. regia, in which the cis-elements were identified. This JVVHAG1 promoter fragment showed expression activity that was enhanced significantly when subjected to the above treatments. Yeast one-hybrid assay and transient expression analysis demonstrated that JrMYB2 specifically bound to the MYBCORE motif and shared similar expression patterns with JVHAGI under $\mathrm{ABA}, \mathrm{CdCl}_{2}$ and $\mathrm{ABA}+\mathrm{CdCl}_{2}$ stress conditions.
\end{abstract}

Conclusions: Our results suggested that the JrVHAG1 gene functions as a $\mathrm{CdCl}_{2}$ stress response regulator by participating in ABA-signal pathway and MYB transcription regulation network. JVHAG1 gene is a useful candidate gene for heavy metal stress tolerance in plant molecular breeding.

Keywords: Heavy metal stress, JVVHAG1 gene, ABA-signal pathway, Promoter, MYB transcription factor

\footnotetext{
*Correspondence: zyl8291290@163.com; yangguiyan@yahoo.com

${ }^{1}$ Hunan Research Center of Engineering Technology for Utilization of

Environmental and Resources Plant, Central South University of Forestry and

Technology, 498 Shaoshan South Road, Changsha, Hunan Province 410004,

China

${ }^{4}$ Laboratory of Walnut Research Center, College of Forestry, Northwest A \& F

University, Yangling, Shaanxi 712100, China

Full list of author information is available at the end of the article
} 


\section{Background}

Heavy metals include micronutrients (such as Fe, Mn, and Mo), trace elements (such as $\mathrm{Cu}, \mathrm{Zn}, \mathrm{Ni}$, and $\mathrm{W}$ ), and stress factors (such as $\mathrm{Cd}, \mathrm{Pb}, \mathrm{Hg}, \mathrm{Ag}$, and $\mathrm{U}$ ) that are toxic to plants [1]. Heavy metal stress not only leads to decrease in plant seed germination, plant growth, or increase of reactive oxygen species (ROS) accumulation and cell death but also induces chlorosis, necrosis, and turgor loss, even modifies the protein profile [2, 3]. More importantly, enrichment of heavy metals in plants and animals causes serious harm to the food chain and human health. Therefore, adequate understanding of the regulatory mechanism and screening the vital functions of genes from corresponding plants are vital to understand plant heavy metal stress responses.

Among the stress related heavy metals, $\mathrm{Cu}, \mathrm{Zn}, \mathrm{Pb}, \mathrm{Ni}$, $\mathrm{Se}, \mathrm{Cr}$, and $\mathrm{Co}$ were moderately enriched, whereas $\mathrm{Sb}$ and cadmium $(\mathrm{Cd})$ were extremely highly enriched in the majority of soil samples, therefore, $\mathrm{Cd}$ is regarded as one of the most phytotoxic heavy metals $[4,5]$. In soil, $\mathrm{Cd}$ contamination could severely affect the performance of agricultural fields [6]. Cd toxicity in agricultural soil has received significant attention because of its high penetration in the food chain and its toxicity to humans [7]. Thus, characterization the molecular mechanisms of potential Cd stress response genes is crucial $[8,9]$.

Several studies on Cd stress-related genes and mechanisms are available, for instance, the phytochelatin synthase 2 gene (OsPCS2) from Oryza sativa improved $\mathrm{Cd}$ and As stress tolerance by mitigating the accumulation of these heavy metals in rice plants [10]. The F-box protein PP2-B15 and zinc transporter 4 from Solanum lycopersicum are indicators of soil Cd contamination as confirmed by transcriptional analyses [7]. The sensitivity of two nearisogenic Triticum aestivum lines with differences at the Rht-B1 locus, Rht-B1a (tall wild type, encoding DELLA proteins), and Rht-B1c (dwarf mutant, encoding modified DELLA proteins) to $\mathrm{Cd}$ stress was investigated. The results suggest that the Rht-B1c-encoded DELLA proteins enhanced Cd tolerance by participating in the photosynthetic apparatus [11]. Heavy metal ATPase 3 (HMA3), a $\mathrm{P}_{1 \mathrm{~B} 2}$-ATPase, is a key tonoplast transporter involved in mediating the vacuolar sequestration of $\mathrm{Cd}$ to detoxify the intake of this element by plants. The HMA3 from Festulolium loliaceum (FlHMA3) plays an important role in $\mathrm{Cd}^{2+}$ sequestration in root cell vacuoles, thereby limiting the entry of $\mathrm{Cd}^{2+}$ into the cytoplasm and reducing $\mathrm{Cd}^{2+}$ toxicity [12]. Vacuolar $\mathrm{H}^{+}$-ATPase (V-ATPase) and its subunits play important roles in heavy metal stress response, and the c subunit from Tamarix hispida (ThVHAc1) confers plants with enhanced $\mathrm{CdCl}_{2}$ stress tolerance through WRKY transcription factor and ROS scavenging [5].

$\mathrm{V}$-ATPase is a multi-subunit complex comprising domains $\mathrm{V}_{1}$ (600-650 kDa membrane-peripheral domain) and $\mathrm{V}_{0}$ (260 kDa membrane-integral domain). The $\mathrm{V}_{1}$ domain contains eight different subunits $(\mathrm{A}-\mathrm{H})$ and is responsible for ATP hydrolysis, while the $V_{0}$ domain includes six different subunits (a, d, c, c', c", and e) and is responsible for proton translocation [13]. Some subunits of the V-ATPase were previously characterized, such as Malus domestica A subunit (MdVHA-A) [14], T. aestivum (RH8706-49) B subunit (TaVB) [15], Pennisetum glaucum c subunit (PgVHA-c1) [16], and Arabidopsis thaliana VHA-B, $-\mathrm{E},-\mathrm{G}$, and -a subunits [17]. Therefore, understanding the abiotic stress response function and the mechanism of V-ATPase as well as its subunits sounds important.

Juglans regia is a nut tree cultivated worldwide for its nutritious fruits [18], whose production is limited by various environmental stimuli. Studies on the stress response mechanism of walnut trees are currently lacking; therefore, achieving a better understanding of the mechanisms involved in abiotic stress response of J. regia is timely and essential [19]. In previous studies, we identified a few candidate genes in walnut involved in stress response, including the $\mathrm{G}$ subunit (JVHAG1), which is a known drought-inducible osmotic stress gene and correlated with the ABA (abscisic acid)-signal pathway [19]. In this study, we found that the expression of JrVHAG1 was induced by $\mathrm{CdCl}_{2}$ stress in J. regia leaves and roots, and the function of $\mathrm{JVVHAG1}$ exposed to $\mathrm{CdCl}_{2}$ treatment was further verified. Meanwhile, the expression activities of JrVHAG1 promoter segment and upstream regulatory genes were analyzed, which suggested a potential $\mathrm{CdCl}_{2}$ response mechanism of JrVHAG1 involving in ABA-signal pathway and MYB transcription regulation.

\section{Results}

Expression of J. regia V-ATPase subunits and response to $\mathrm{CdCl}_{2}$ and $\mathrm{ABA}$ treatments

To better understand the role of V-ATPase subunits in abiotic stress response in walnut tree, $15 \mathrm{~V}$-ATPase subunits were identified from the $J$. regia transcriptome in tissues using functional annotation of non-redundant unigenes (NRUs), which were analyzed by blast and denoted as VHA-A, B, C, D, E, F, G (JrVHAG1), H, a1, a3, $\mathrm{c} 1, \mathrm{c} 4, \mathrm{~d} 1, \mathrm{~d} 2$, and $\mathrm{e} 1$, respectively. Transcription levels of these subunits under $\mathrm{CdCl}_{2}, \mathrm{ABA}$, and $\mathrm{ABA}+\mathrm{CdCl}_{2}$ $\left(\mathrm{CdCl}_{2}\right.$ plus $\left.\mathrm{ABA}\right)$ were analyzed by quantitative real time PCR (qRT-PCR) in the roots and leaves of J. regia. The results showed that most of these subunits were induced by $\mathrm{CdCl}_{2}$ and $\mathrm{ABA}$ treatments (Fig. 1). In leaves, the expression patterns upon exposure to $\mathrm{ABA}, \mathrm{CdCl}_{2}$, $\mathrm{ABA}+\mathrm{CdCl}_{2}$ could be classified into four groups. VHA$\mathrm{A}$ and $\mathrm{e} 1$ had the maximum transcription under $\mathrm{CdCl}_{2}$, but had the minimum under ABA stress; While VHA-D, E, F, H, a1, a3, d1 subunits were grouped together based 

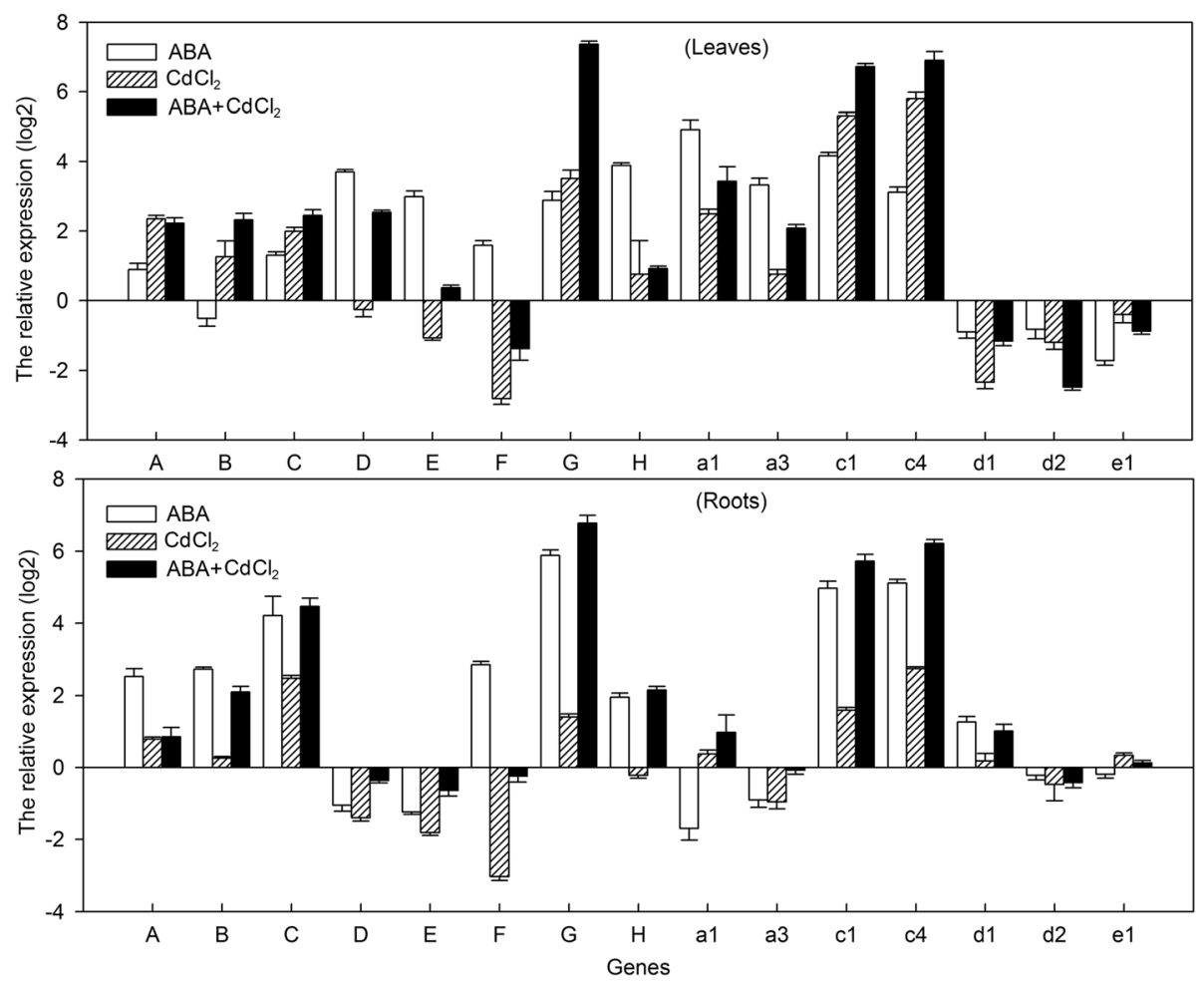

Fig. 1 The expression of JVHAGI in J. regia under ABA and $\mathrm{CdCl}_{2}$ stress. The relative expression level = transcription level under stress treatment/ transcription level under control condition (watered well by fresh water at the same time point as stress treatment). Error bars were obtained from three replicates of qRT-PCR

on their expression patterns that were contrary to VHA$\mathrm{A}$ and $\mathrm{e} 1$; they hit a peak upon exposure to ABA, but declined to bottom upon exposure to $\mathrm{CdCl}_{2}$ stress. The expression levels of VHA-B, C, JrVHAG1, c1 and c4 subunits were increased for $\mathrm{ABA}$ and $\mathrm{ABA}+\mathrm{CdCl}_{2}$ stress, whose transcription was up-regulated upon addition of ABA to $\mathrm{CdCl}_{2}$ treatments, while the VHA-d2 subunit gene was down-regulated by all the stresses, and the tendency was reverse to the levels of VHA-B, C and G (Fig. 1). Under ABA treatment, the VHA-D, E, G, H, a1, a3, $\mathrm{c1}$, c4 were induced to 7.38- 30.20-fold of the control; VHA-B, d1, d2, e1 were suppressed. Under $\mathrm{CdCl}_{2}$ stress, VHA-D, E, F, d1, d2 and e1 subunits were downregulated, among which the $\mathrm{F}$ subunit was the least, whereas VHA-G, c1 and $c 4$ were the top induced three genes. When treated with $\mathrm{ABA}+\mathrm{CdCl}_{2}$, VHA-G, $\mathrm{c} 1$ and c4 were induced to 165.42-, 105.42-, 119.43-folds of the control, respectively. Moreover, the transcription of JrVHAG1 was increased much more obviously by ABA $+\mathrm{CdCl}_{2}$ than all other subunits (Fig. 1).

In the roots, the expression profiles of most subunits exposed to $\mathrm{ABA}, \mathrm{CdCl}_{2}, \mathrm{ABA}+\mathrm{CdCl}_{2}$ were different from those seen in the leaves, excluding VHA-F, d1 and e1 (Fig. 1). According to their transcription patterns, these subunits could also be divided into four groups. Group 1 contained VHA-A, B, F, d1, and d2, whose expression reached a peak when subjected to ABA treatment, while reached the least when subjected to $\mathrm{CdCl}_{2}$ stress. The VHA-e1 subunit formed a separate group for its specific expression pattern. VHA-a1 was also by itself in a class as the expression was highest when exposed to $\mathrm{ABA}+\mathrm{CdCl}_{2}$, while it became the lowest when subjected to ABA. The other subunits were grouped as one family, whose maximum transcription was noticed when treated with $\mathrm{ABA}+\mathrm{CdCl}_{2}$, while the minimum occurred when subjected to $\mathrm{CdCl}_{2}$ stress (Fig. 1). Under ABA treatment, the expression of VHA-C, G, c1, c4 were induced much more obviously than others, and the expression values were 18.55- 58.89-fold of the control. The VHA-D, E, a1, a3 were all down-regulated to lower levels compared to the other subunits. Under $\mathrm{CdCl}_{2}$ stress, the VHA-F subunit was strongly suppressed. The VHA-C, G, c1, c4 were up-regulated to 2.65- 6.65-fold of the control. When treated with $\mathrm{ABA}+\mathrm{CdCl}_{2}$, VHA-C, G, c1, c4 were strongly induced to a much higher level than other subunits, and the highest transcription level was JrVHAG1 (110.13-fold of those of the control) (Fig. 1).

\section{Overexpression of JrVHAG1 improves plant $\mathrm{CdCl}_{2}$ stress tolerance}

For a complete characterization of the $\mathrm{CdCl}_{2}$ stress tolerance of JrVHAG1 gene, whether it involves the ABA- 

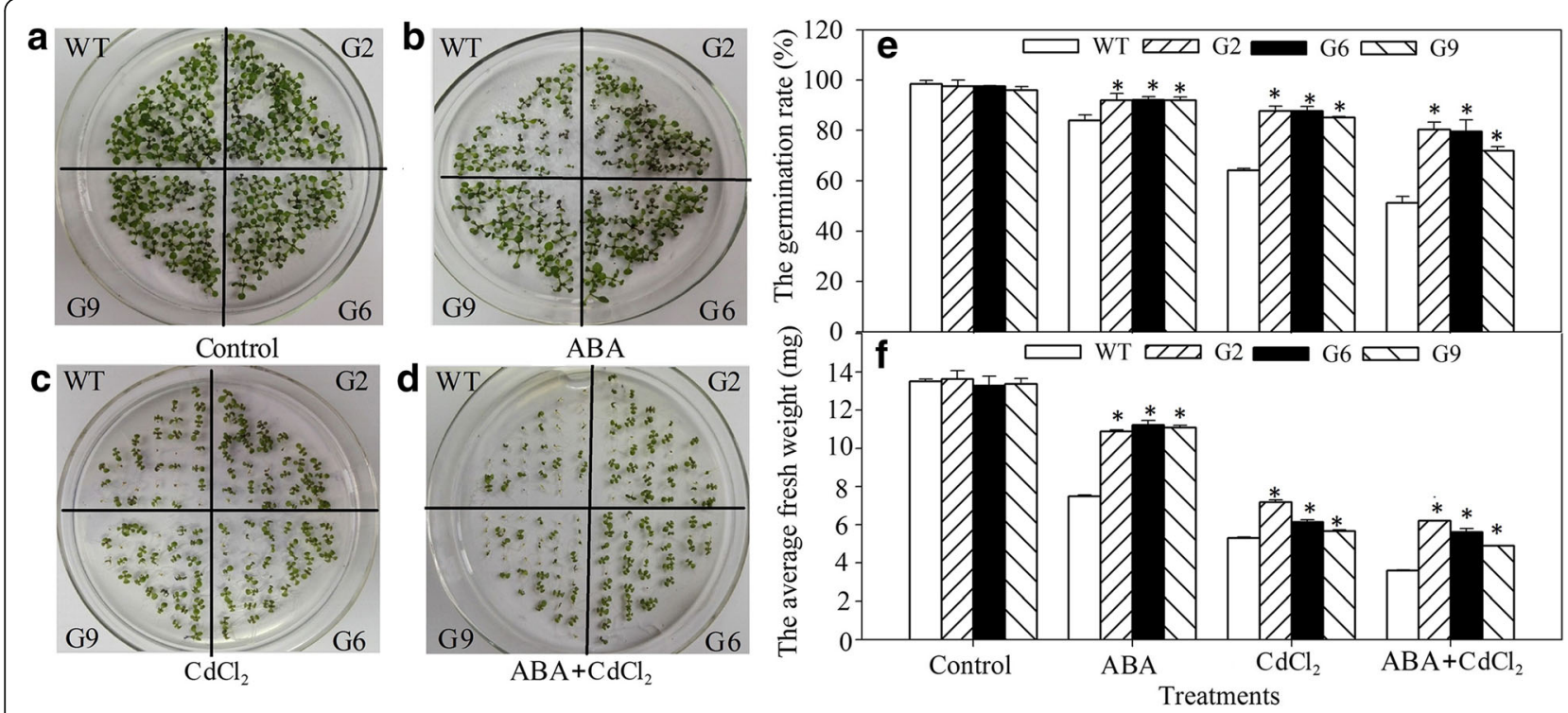

Fig. $2 \mathrm{CdCl}_{2}$ stress tolerance analysis of wild type plants and the transgenic Arabidopsis. WT, wild type; G2, G6, G9 are three JrVHAG1 transgenic lines. a-d, germination under normal condition, $\mathrm{ABA}, \mathrm{CdCl}_{2}$ and $\mathrm{ABA}+\mathrm{CdCl}_{2}$ treatments, accordingly; e, germination percentage of WT, G2, G6, and G9 according to $\mathbf{a}-\mathbf{d}$; $\mathbf{f}$, average fresh weight of the germinated seedlings from a-d. All data are displayed as the mean \pm S.D. of three independent experiments. ${ }^{*}$ means the differences between WT and transgenic seedlings were significant $(p<0.05)$

signal pathway, the germination, biomass, ROS metabolism, and physiological performance of three transgenic lines that overexpression of JrVHAG1 (G2, G6, and G9) [19] and wild type (WT) Arabidopsis were analyzed when exposed to $\mathrm{CdCl}_{2}, \mathrm{ABA}$ and $\mathrm{ABA}+\mathrm{CdCl}_{2}$. The results showed that the germination rates of G2, G6, and G9 were significantly higher $(p<0.05)$ than that of WT under the three treatments, which averaged 1.10-, 1.35-, and 1.51-fold increase compared to that of the WT under $\mathrm{CdCl}_{2}, \mathrm{ABA}$, and $\mathrm{ABA}+\mathrm{CdCl}_{2}$, respectively (Fig. 2a-e). The average fresh weight of the germinated seedlings of G2, G6, G9 were 1.48-, 1.19-, 1.55-folds higher than those of WT when subjected to the above treatments, accordingly (Fig. 2f). When 8-day-old seedlings of WT, G2, G6, and G9 grown on 1/2 MS were transferred to $1 / 2 \mathrm{MS}$ with or without $\mathrm{ABA}, \mathrm{CdCl}_{2}$, $\mathrm{ABA}+\mathrm{CdCl}_{2}$ and cultured for additional $8 \mathrm{~d}$, the fresh weight and primary root length were similar among the four lines under control conditions. However, the biomass accumulation (fresh weight and primary root length) of the transgenic lines were significantly higher $(p<0.05)$ than that of WT (Fig. 3a-d). The average fresh weight of G2, G6, and G9 lines were 1.26- $\left(\mathrm{CdCl}_{2}\right)$ and 1.34-folds $\left(\mathrm{ABA}+\mathrm{CdCl}_{2}\right)$ of that of WT (Fig. 3e). Growth of the primary roots of the WT were severely affected by the three treatments, among which the most serious inhibition was happened on $\mathrm{ABA}+\mathrm{CdCl}_{2}$ stress (Fig. 3f). These results suggest that overexpression of $J V V H A G 1$ plays a positive role in the response to $\mathrm{CdCl}_{2}$ stress in plants.
To further verify the $\mathrm{CdCl}_{2}$ tolerance role of JrVHAG1, 47-d-old WT and transgenic plants were treated with $\mathrm{ABA}$, or $\mathrm{CdCl}_{2}$, or $\mathrm{ABA}+\mathrm{CdCl}_{2}$. 3, 3'-Diaminobenzidine (DAB) and nitrogen blue tetrazolium (NBT) staining clarified that the WT seedlings were stained deeper than those of G2, G6, and G9 when exposed to all above three treatments (Fig. 4a, b). The hydrogen dioxide $\left(\mathrm{H}_{2} \mathrm{O}_{2}\right)$ accumulation in the WT plants was also significantly higher than those seen in the transgenic plants (Fig. 4c). Furthermore, the electrolyte leakage (EL) rates of these lines had a trend similar to that as $\mathrm{H}_{2} \mathrm{O}_{2}$. Under ABA treatment, the EL rates of G2, G6, G9 were $69.28 \% \sim 73.57 \%$ of that of WT; but when exposed to $\mathrm{CdCl}_{2}$, the differences were much more obvious compared to those under $\mathrm{ABA}$ stress and they were $45.14 \% \sim 63.98 \%$ of that of WT; When treated with ABA $+\mathrm{CdCl}_{2}$, the corresponding rates were $49.57 \% \sim 69.13 \%$ of that of WT, respectively (Fig. 4d). The superoxide dismutase (SOD) and peroxidase (POD) activities, proline and malondialdehyde (MDA) contents were also supportive of the role of JrVHAG1 that it could effectively improve plant tolerance to $\mathrm{CdCl}_{2}$ (Fig. 5). Upon exposure to $\mathrm{ABA}, \mathrm{CdCl}_{2}, \mathrm{ABA}+\mathrm{CdCl}_{2}$ stresses, the MDA contents of the tested lines were similar to that of the EL rates and $\mathrm{H}_{2} \mathrm{O}_{2}$ contents, WT had higher values, 1.241.75-fold, 1.19- 1.67-fold and 1.16- 1.64-fold of transgenic seedlings, respectively. However, the SOD, POD and proline levels were contrasting with MDA levels that the SOD, POD and proline levels of three transgenic 


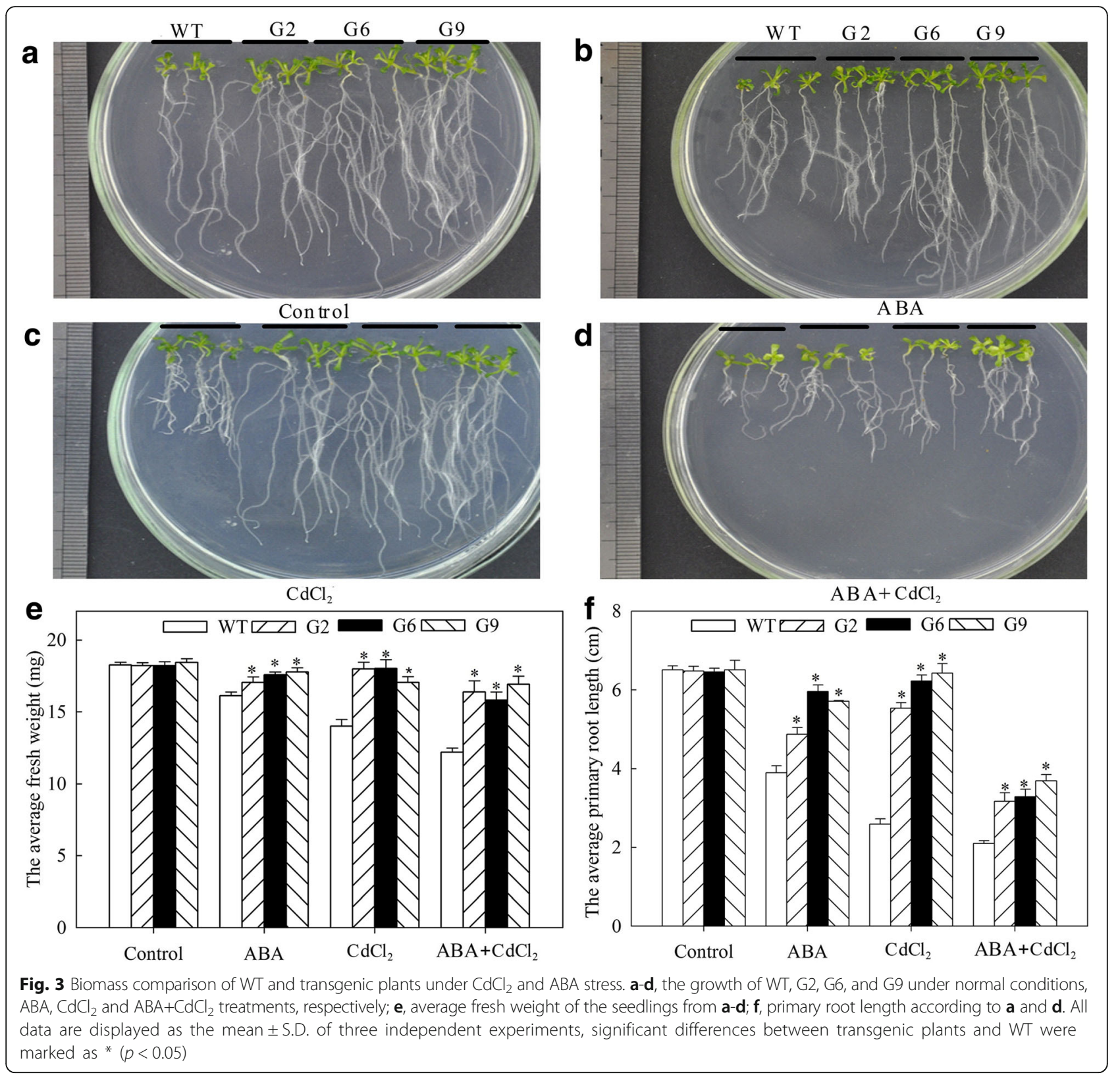

lines were 2.08- 2.75-fold, 1.32- 1.60-fold and 1.311.45 -fold of that of WT, accordingly (Figs. 4, 5).

\section{Identification and expression activity of JrVHAG1 promoter}

A 1200 bp promoter segment of JrVHAG1 was identified from the J. regia genome that was located in the 538,241534,657 region of the walnut genome (NW017389860.1). The promoter sequence and the relevant elements such as, ARE, LTR, MYBCORE, W-box are shown in Additional file 1: Figure S2. This promoter fragment was inserted into a $\beta$-glucuronidase (GUS) expression vector pCAMBIA1301, and Arabidopsis plants were transformed using this vector. GUS staining revealed that the promoter caused GUS expression in the leaves and roots (Fig. 6). When treated with $\mathrm{ABA}, \mathrm{CdCl}_{2}$, and $\mathrm{ABA}+\mathrm{CdCl}_{2}$, the transgenic plants containing this promoter showed increased GUS activity overall in the aerial parts and roots compared to control. The total GUS activities exposed to $\mathrm{ABA}, \mathrm{CdCl}_{2}$, and $\mathrm{ABA}+\mathrm{CdCl}_{2}$ stresses were 1.22-, $1.30-$, and 1.49-fold higher of that under normal condition, correspondingly, and the differences were significant (Fig. 6).

\section{Up-stream regulation of JrVHAG1 involves MYB transcriptional activation}

To verify the up-stream regulation of JrVHAG1, yeast one-hybrid assay was employed to study the interactions 


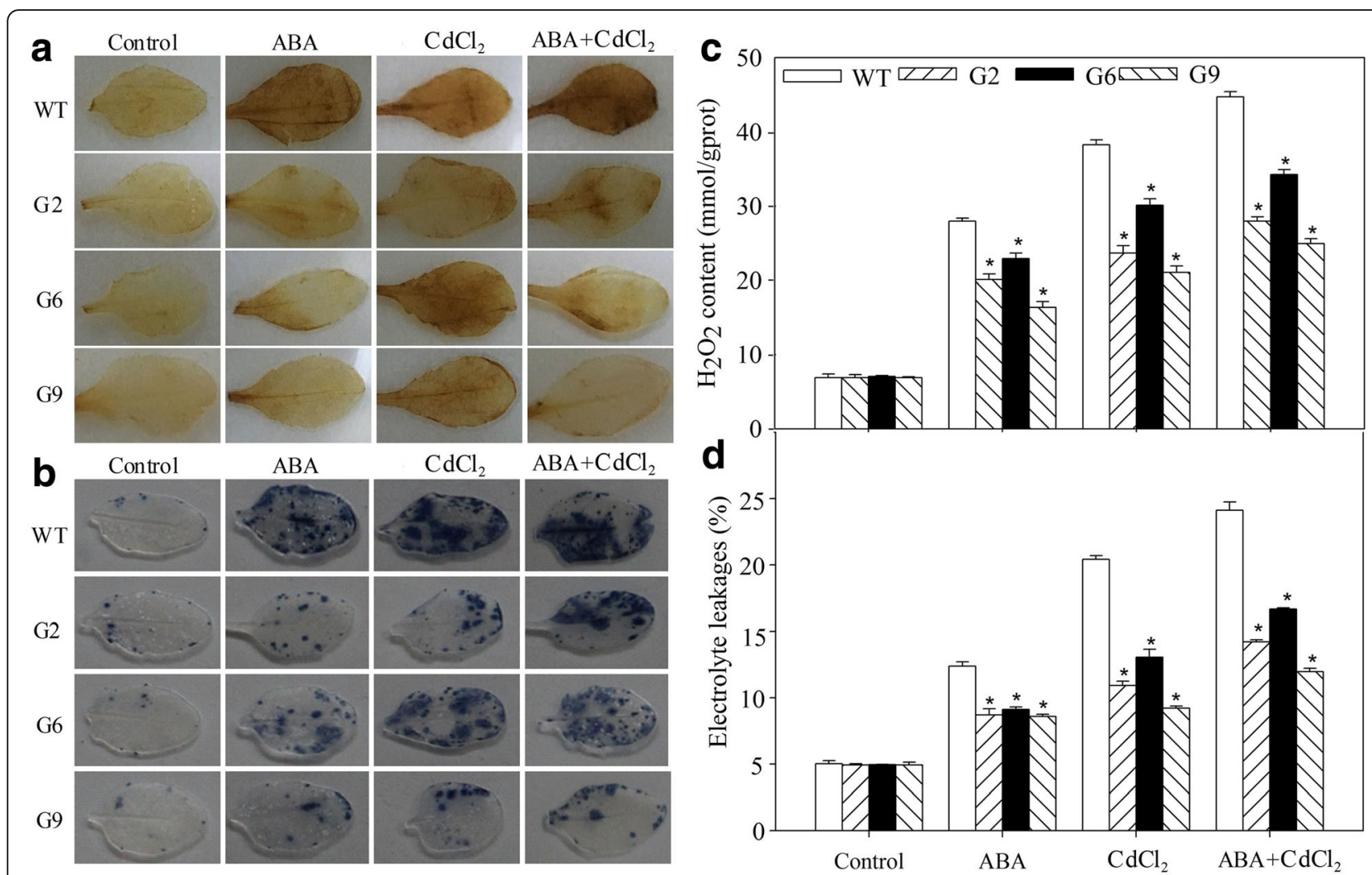

Fig. 4 ROS levels in transgenic plants and WT under $\mathrm{CdCl}_{2}$ and $A B A$ stress. $\mathbf{a}$, DAB staining; $\mathbf{b}$, NBT staining; $\mathbf{c}, \mathrm{H}_{2} \mathrm{O}_{2}$ content; $\mathbf{d}$, EL rate. All experiments were repeated at least three times, and approximately 15 samples collected from multiple seedlings in each experiment. All data are displayed as the mean \pm S.D. of three independent experiments, significant differences between transgenic lines and WT $(p<0.05)$ were indicated by *

between transcription factors (TFs) and MYBCORE in the promoter. It was found that $J r M Y B 2$ binds to the MYBCORE motif, which was confirmed by the interaction between pHis2-MYB-M (mutated MYBCORE), pHis2-MYB-S (the JrVHAG1 promoter including the MYBCORE motif), pHis2-MYB-M1 (the JrVHAG1 promoter excluding the MYBCORE motif), or pHis2-MYBM2 (the JrVHAG1 promoter including the mutated MYBCORE motif) and JrMYB2 on the SD (synthetic drop-out medium)/-Trp-Leu-His/50 mM 3-AT (3amino-1, 2, 4-triazole) solid medium (Fig. 7a). Moreover, the co-transformation of the reportor and effector demonstrated that the GUS activities of the leaves that were transformed by the MYBCORE motif or the promoter fragments containing the MYBCORE motif were similar to that of the positive control, and significantly higher than those of the negative control and mutated reportors. The GUS activities of leaves transformed by effector and the mutated reportor was similar to that of the negative control, further suggesting that JrMYB2 binds specifically to the MYBCORE motif in the JrVHAG1 promoter (Fig. 7b).

The results of qRT-PCR analysis of JrMYB2 clearly showed that $\operatorname{JrMYB2}$ was induced by $\mathrm{ABA}, \mathrm{CdCl}_{2}$, and
$\mathrm{ABA}+\mathrm{CdCl}_{2}$ in the leaves and roots (Fig. 7c). In leaves, the transcription of JrMYB2 was induced by 3.97-, 7.01-, and 12.04-fold of control when subjected to $\mathrm{ABA}$, $\mathrm{CdCl}_{2}$, and $\mathrm{ABA}+\mathrm{CdCl}_{2}$ stresses, and the corresponding gene expression in the roots were 11.96-, 3.34-, and 15.14-fold compared to control, respectively (Fig. 7c). It appears that the expression profiles of JrMYB2 under these stresses in both tissues are similar to those of JrVHAG1, especially when subjected to the same conditions (Fig. 1), indicating that $J r M Y B 2$ may act as an upstream regulator of JrVHAG1 to either control JrVHAG1 or act along with $J r V H A G 1$ to improve $\mathrm{CdCl}_{2}$ stress tolerance of plants.

\section{Discussion}

The V-ATPase regulates and is also self-regulated by various signaling cascades controlling nutrient supply and metabolism [20]. The up-regulation of the VATPase subunits is beneficial to plants as part of the heavy metal stress response. For instance, Cucumis sativus CsVHA-c1, CsVHA-c2, and CsVHP1;1 are essential elements of the mechanisms involved in the adaptation of cucumber plants to copper toxicity [21]. T. hispida c subunit (ThVHAc1) was up-regulated by $\mathrm{CdCl}_{2}$ stress, 

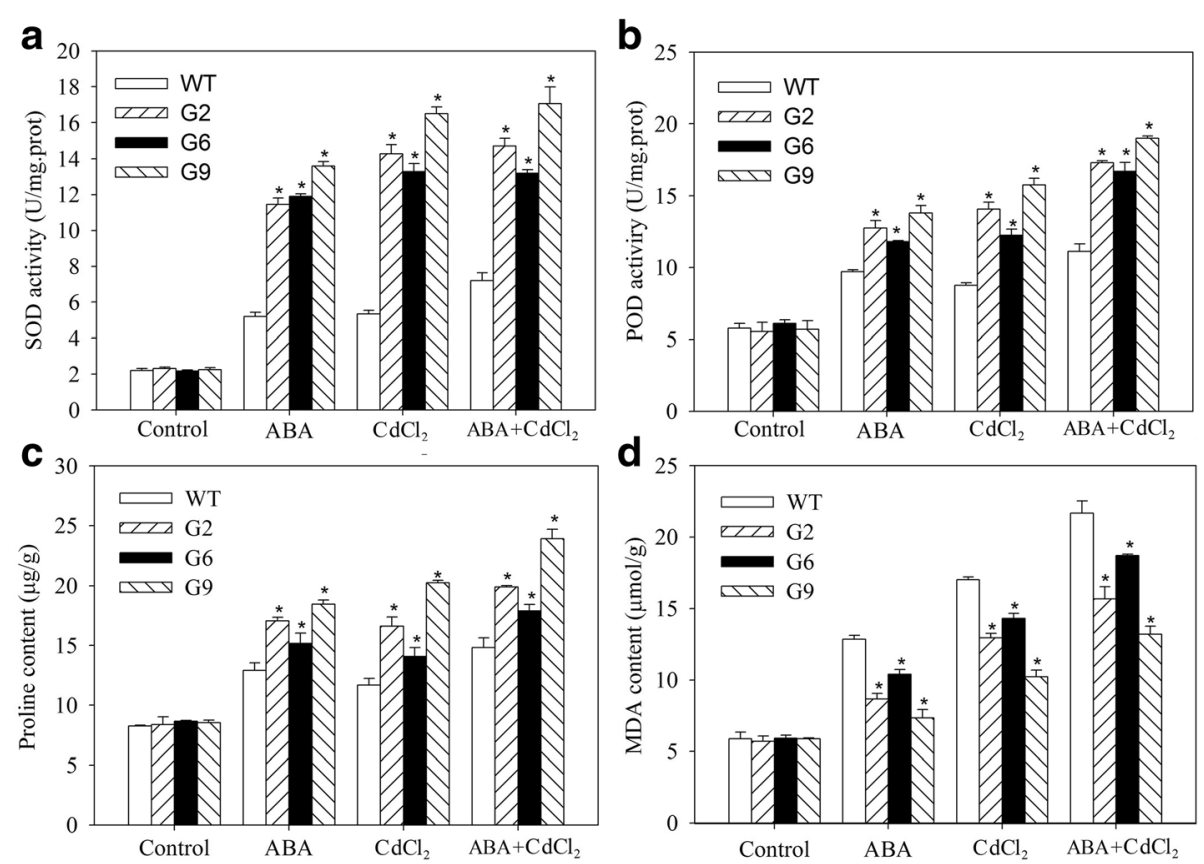

Fig. 5 Physiological index analysis of JrVHAG1 transformed seedlings under $\mathrm{CdCl}_{2}$ and ABA stress. a, SOD activity; b, POD activity; c, proline content; $\mathbf{d}$, MDA content. Every experiment was repeated three times. All data are displayed as the mean \pm S.D. of three independent experiments. ${ }^{*}$ means significant differences $(p<0.05)$ between WT and transgenic seedlings

and the overexpression could effectively improve the $\mathrm{CdCl}_{2}$ tolerance of plants [5]. In a previous study, we demonstrated that the involvement of JrVHAG1 in drought-inducible osmotic stress correlated with ABAsignal pathway [19], implying the potential response mechanism of $J$. regia $\mathrm{V}$-ATPase and its subunits involve in ABA-signal regulation. To well understand the adaptation mechanism of $J$. regia to heavy metal stress, in this study, we firstly identified $15 \mathrm{~V}$-ATPase subunits and their expression were analyzed by qRT-PCR under $\mathrm{CdCl}_{2}$ and ABA treatments. The results showed that the expression of VHA-B, C, JrVHAG1, c1 and c4 subunits were similar and induced much more obviously than others exposed to the three treatments, whose transcription was up-regulated more obviously by $\mathrm{ABA}+\mathrm{CdCl}_{2}$ than single stress of $\mathrm{ABA}$ or $\mathrm{CdCl}_{2}$ (Fig. 1), suggesting the possibly positive role of $V H A-B, C$, JrVHAG1, $c 1$ and $c 4$ to $\mathrm{CdCl}_{2}$ stress and may involve in ABA-signal pathway. To understand rapidly of the prediction, the open reading frames (ORFs) of VHA-B, C, JrVHAG1, $c 1$ and $c 4$ were inserted into the pYES2 and transformed into INVSC1, respectively [22]. Then the transgenic yeasts were treated with $\mathrm{ABA}, \mathrm{CdCl}_{2}, \mathrm{ABA}+\mathrm{CdCl}_{2}$, and compared to the control yeast. The growth abilities of $V H A$ $B, C, J r V H A G 1, c 1$ and $c 4$ transformed yeasts were higher than that of the control yeast. The most important is that the JrVHAG1 transformed yeasts showed the best growth activities under the three treatments, and the differences between JrVHAG1 transgenic yeast and others were significant under $\mathrm{ABA}+\mathrm{CdCl}_{2}$ stress (Additional file 1: Figure S1), indicating that the ABA truly promoted the function of $\operatorname{JVHAG1}$ in $\mathrm{CdCl}_{2}$ tolerance. Considering that the expression of JrVHAG1 was highly induced by $\mathrm{CdCl}_{2}$ stress and was enhanced to a very high level by $\mathrm{ABA}+\mathrm{CdCl}_{2}$ (Fig. 1), we think JrVHAG1 was a potential candidate gene for $\mathrm{CdCl}_{2}$ stress tolerance and was likely to connect with ABA signal.

To verify the function of JrVHAG1 in Cd stress, transgenic Arabidopsis lines with high expression of this gene were evaluated. The results showed that the germination rates, biomass accumulations, and protective enzyme activity of the transgenic lines were significantly higher than those of the WT plants; while the ROS generation of G2, G6, and G9 were significantly lower than that of WT (Figs. 2, 3, 4, 5) and perhaps indicating the JrVHAG1 gene functions in ROS scavenging, suggesting that the expression of JrVHAG1 could improve plant Cd stress tolerance. These physiological index endowing by JrVHAG1 under $\mathrm{ABA}, \mathrm{CdCl}_{2}$ and $\mathrm{ABA}+\mathrm{CdCl}_{2}$ treatments were similar to other subunits those were verified to be abiotic stress tolerance genes. Such as $M$. domestica MdVHA-A and Puccinellia tenuiflora PtVHAc. Overexpression of MdVHA-A conferred transgenic tobacco seedlings with enhanced drought tolerance by improving important attributes such as dry weight, fresh weight, MDA, and relative water content [14]. Transgenic A. thaliana lines expressing PtVHAc exhibited improved tolerance to salt-induced osmotic stress as 


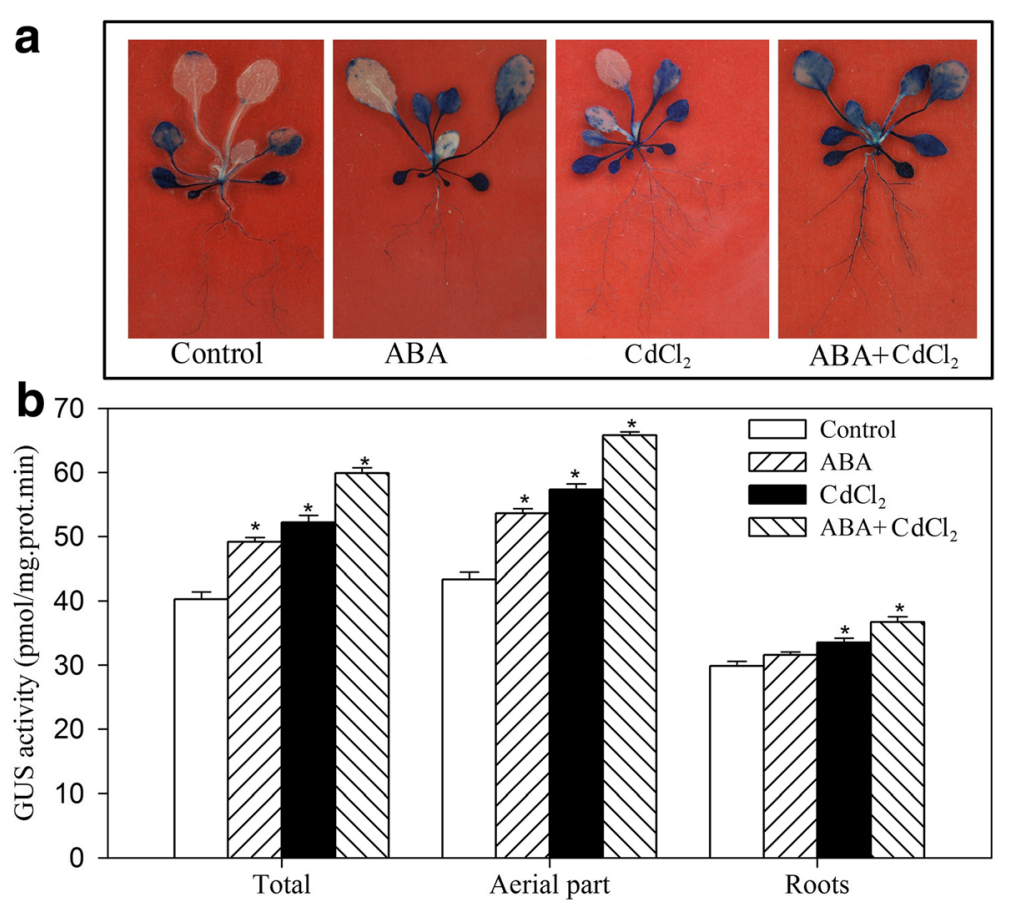

Fig. 6 The expression activity of JVVHAG1 promoter. a, GUS staining of the JrVHAG1 promoter transgenic plants under normal, $A B A, C d C l_{2}, A B A$ $+\mathrm{CdCl}_{2}$ conditions. $\mathbf{b}$, the GUS activities according to $\mathbf{a}$. Every experiment was repeated three times. All data are displayed as the mean \pm S.D. of three independent experiments. * means significant differences $(p<0.05)$ between the stress conditions and normal condition

revealed from the analysis of the fresh weight, root length, silique number, and other parameters [23]. Moreover, $\mathrm{ABA}$ is known as a stress hormone that takes part in the integration of signals [24]. Germination and plant growth under heavy metal stress is related to ABA $[25,26]$. The current results also revealed that the difference of germination rates and average fresh weight between the transgenic lines and WT under $\mathrm{ABA}+\mathrm{CdCl}_{2}$ stress was significant than that exposed to $\mathrm{CdCl}_{2}$ (Fig. 2), implying that the ABA promotes the function of JrVHAG1 gene in response to $\mathrm{CdCl}_{2}$ stress, which were just similar to other previous reports. For example, after exposure to $\mathrm{CdCl}_{2}$ stress, the S. lycopersicum Micro-Tom (MT) sitiens ABAdeficient mutant (sit) and its WT counterpart displayed differences in lipid peroxidation, hydrogen peroxide content, and activities of some key antioxidant enzymes such as catalase, glutathione reductase, and ascorbate peroxidase, highlighting the relative importance of $\mathrm{ABA}$ in $\mathrm{Cd}$ stress response of plants [24]. The basic helix-loop-helix (bHLH) in tartary buckwheat (Fagopyrum tataricum) (FtbHLH3) was induced by polyethylene glycol 6000 $\left(\mathrm{PEG}_{6000}\right)$ and $\mathrm{ABA}$ treatment. Overexpression of FtbHLH3 in Arabidopsis resulted in increased drought/ oxidative tolerance and indicates that FtbHLH3 may function as a positive regulator of drought/oxidative stress tolerance through an ABA-dependent pathway [27]. These results told us that $\mathrm{JVVHAG1}$ function as a positive $\mathrm{CdCl}_{2}$ response gene involved in ABA-signaling pathway.
In plants, transcription factors are important components of stress response pathways involving in many stress-response genes. There are several studies on the function of Cd-responsive genes; however, the upstream transcriptional regulatory pathways that modulate their responses to $\mathrm{Cd}$ are less clear [28]. To better understand the Cd stress response mechanism of JrVHAG1, the promoter of JrVHAG1 was identified and the expression activity was tested. The promoter displayed different expression activities in the roots and leaves under normal and $\mathrm{CdCl}_{2}$ treated conditions (Fig. 6), which was similar to the expression of JrVHAG1 upon exposure to $\mathrm{CdCl}_{2}$ stress (Fig. 1), indicating the effectiveness of the selected promoter fragment. Further, the up-stream regulator of JrVHAG1 was identified through yeast onehybrid assay and co-transient expression experiments. JrMYB2 was found to specially bind to the MYBCORE motif present in the JrVHAG1 promoter, and the expression of $J r M Y B 2$ was similar to that of JrVHAG1 under $\mathrm{ABA}, \mathrm{CdCl}_{2}$, and $\mathrm{ABA}+\mathrm{CdCl}_{2}$ treatments (Fig. 7), indicating that $J r M Y B 2$ acts as an up-stream regulator of JrVHAG1 and could regulate or combine functionality with $\mathrm{JVVHAG1}$ in $\mathrm{CdCl}_{2}$ stress response. This prediction is similar to that in other studies. For instance, T. hispida ThWRKY7, the up-stream regulator of ThVHAc1, regulates plant $\mathrm{CdCl}_{2}$ tolerance and controls the involvement of ThVHAc1 in plant $\mathrm{CdCl}_{2}$ stress response by binding to the WRKY motif present in the ThVHAc1 

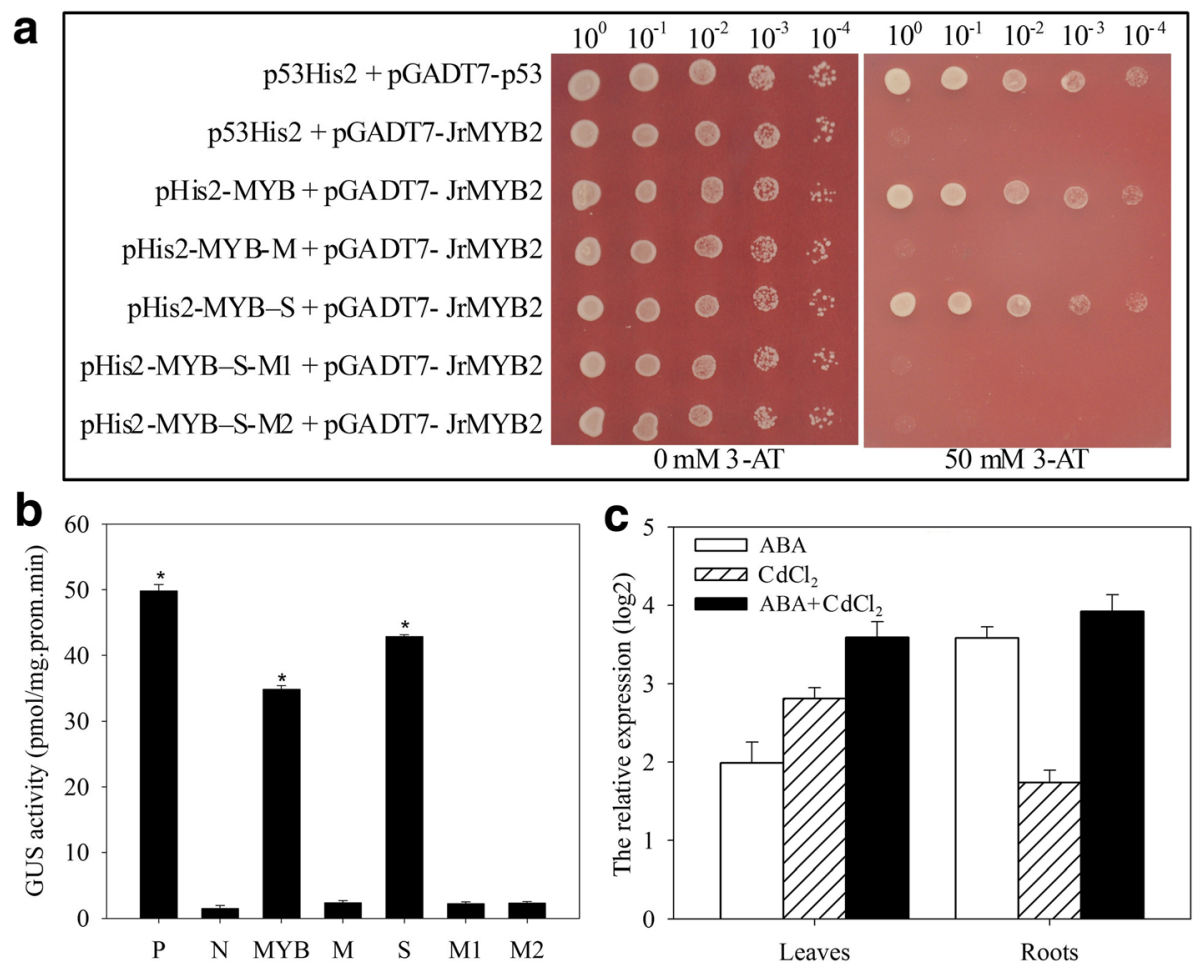

Fig. 7 Analysis of the up-steam regulators of JrVHAG1. a, yeast one-hybrid assay analyses of the upstream regulators of JrVHAG1. p53His2 + pGADT7p53, positive control, pGADT7-Rec2 vector that encodes murine p53 fused with GAL4 AD; p53His2 + pGADT7-JrMYB2, negative control, pHis2 reportor vector that contains the cis-acting DNA consensus sequence recognized by p53. The transformants spotted on SD/-His/-Leu/-Trp plates with 0 mM 3-AT were used as positive controls for transformant growth. Positive transformants were further confirmed by spotting serial dilutions (1/1, 1/10, 1/ 100, 1/1000, 1/10000) onto SD/-His/-Leu/-Trp plates with $50 \mathrm{mM}$ 3-AT. $\mathbf{b}$, transient co-transformation of the reportor and effectors into tobacco seedlings, the GUS activities was determined. P, positive control; N, negative control, MYB, M, S, M1, M2 were consistent with a. * means significant differences $(p<0.05)$ between the negative control and others. $\mathbf{c}$, the expression of JrMYB2 under $\mathrm{ABA}, \mathrm{CdCl}, \mathrm{ABA}+\mathrm{CdCl}_{2}$ treatments. Every experiment was repeated three times. All data are displayed as the mean \pm S.D. of three independent experiments.

promoter [5]. Lin et al. demonstrated that the Phaseolus vulgaris ethylene response factor 15 (PvERF15), an upstream transcriptional regulator of the $\mathrm{Cd}$ metal response element-binding transcription factor 1 ( $P v M T F-$ 1) was identified using a yeast one-hybrid system. It was strongly induced by $\mathrm{CdCl}_{2}$ stress and activated the PvERF15/PvMTF-1 transcriptional interaction [28]. These results suggested that the $\mathrm{Cd}$ stress response was controlled by up-stream regulators and yeast one-hybrid assay is an effective way to screen the potential regulatory factors. Meanwhile, MYB TFs are members of a large family that have multiple functions in plant growth and development, flavonoid metabolism, and biotic/abiotic stress responses [29]. In $A$. thaliana, the rootspecific transcription factor $M Y B 72$ is required for the onset of induced systemic resistance (ISR) and is also associated with plant survival under conditions of iron deficiency [30]. Ectopic expression of orchid (Dendrobium sp. XMW-2002-2) R2R3-MYB gene DwMYB2 in Arabidopsis confers hypersensitivity to iron deficiency in the transgenic plants. The ferric-chelate reductase gene, AtFRO2, and the iron transporter genes, AtIRT1 and
AtIRT2, are consistently up-regulated by the expression of $D w M Y B 2$, while other potential iron transporters such as AtIREG1, AtFRD3, and NRAMP1 are down-regulated, indicating the role of $D W M Y B 2$ in iron transportation impairment [31]. SbMYB44, a R2R3-MYB cloned from Salicornia brachiata Roxb., was up-regulated in response to salinity, desiccation, high temperature, and treatments with ABA and salicylic acid (SA). Overexpression of SbMYB44 enhanced the growth of yeast cells under both ionic and osmotic stresses [32]. Considering all these studies and our results, we consider that the JrVHAG1 gene is an important $\mathrm{CdCl}_{2}$ response gene and is involved in MYB transcription pathway.

\section{Conclusion}

The transcription of JrVHAG1 was highly up-regulated by $\mathrm{CdCl}_{2}$ and ABA stress in J. regia roots and leaf tissues. Heterologous overexpression of JrVHAG1 in A. thaliana conferred the transgenic seedlings with increased fresh weight and primary root length, higher SOD and POD activities, as well as lower levels of ROS, $\mathrm{H}_{2} \mathrm{O}_{2}, \mathrm{MDA}$, and $\mathrm{EL}$ rates than those of WT plants 
exposed to $\mathrm{ABA}, \mathrm{CdCl}_{2}, \mathrm{ABA}+\mathrm{CdCl}_{2}$ treatments. A 1200 bp promoter fragment containing many cis-elements of JrVHAG1 was isolated and demonstrated to show high expression activity, which was induced by $\mathrm{CdCl}_{2}$ and $\mathrm{ABA}$ treatments. Yeast one-hybrid and cotransient transformation assays in tobacco showed that JrMYB2 could specifically bind to the MYBCORE motif in the JrVHAG1 promoter. Similar to JrVHAG1, JrMYB2 could also be induced by $\mathrm{CdCl}_{2}$ and $\mathrm{ABA}$. Considering that the clear responses of JrVHAG1 and JrMYB2 to $\mathrm{CdCl}_{2}$ and $\mathrm{ABA}$ treatments in walnut root and leaf tissues and the $\mathrm{CdCl}_{2}$ tolerance conferred by overexpression of JrVHAG1 in transgenic Arabidopsis, it can be concluded that JrVHAG1 plays a positive role in Cd tolerance through ABA-signal pathway and involves in MYB transcription regulation.

\section{Methods}

\section{Plant materials and growth conditions}

New branches from 6-year-old grafted 'Xiangling' seedlings (a genotype of $J$. regia widely planted in China) were obtained and grafted to 2-year-old stocks of the same 'Xiangling'. The grafted seedlings were cultivated for 2-years in a greenhouse $\left(22 \pm 2{ }^{\circ} \mathrm{C}\right.$, relative humidity $70 \pm 5 \%$, illumination cycle $14 / 10 \mathrm{~h}$ ) [33], and treated with $50 \mu \mathrm{M} \mathrm{ABA}$, or $150 \mu \mathrm{M} \mathrm{CdCl}_{2}$, or $150 \mu \mathrm{M} \mathrm{CdCl}_{2}$ plus $50 \mu \mathrm{M} \mathrm{ABA}\left(\mathrm{ABA}+\mathrm{CdCl}_{2}\right)$ by watering the roots for $60 \mathrm{~h}$. The roots and leaves were harvested independently and stored at $-80{ }^{\circ} \mathrm{C}$ for qRT-PCR analysis. The seedlings watered only with fresh water for $60 \mathrm{~h}$ served as control. Every treatment was applied three times and each treatment contained at least 9 seedlings.

\section{RNA isolation and expression analysis of V-ATPase subunits}

V-ATPase subunits were identified from the J. regia transcriptome in tissues using functional NRUs, which were analyzed using BLAST tools. The ORFs were screened using the ORF finder tool (https://www.ncbi.nlm.nih.gov/ orffinder/), and the primers used for qRT-PCR analysis were designed from the 5 '-end of the ORF (Additional file 1: Table S1). Total RNA from each sample was isolated using the cetyltrimethylammonium ammonium bromide (CTAB) method and reverse-transcribed into cDNA [34], which was diluted to $1 / 10$ of the original concentration with sterile water for use as template of qRT-PCR. The $18 \mathrm{~S}$ rRNA was used as an internal control gene [35]. The $20 \mu \mathrm{L}$ reaction mixture contained $2 \mu \mathrm{L}$ cDNA template (equivalent to $100 \mathrm{ng}$ of total RNA), $0.5 \mu \mathrm{M}$ of each forward and reverse primer (Additional file 1: Table S1), $10 \mu \mathrm{L}$ of SYBR Green Real-time PCR Master Mix (CWBIO). The qRT-PCR was performed in a CFX96 Touch $^{\text {Tx }}$ Real-Time PCR Detection System (Bio-Rad Laboratories, Redmond, WA) [33]. The following cycling parameters were applied for amplification: $94{ }^{\circ} \mathrm{C}$ for $30 \mathrm{~s}$ followed by 44 cycles at $94{ }^{\circ} \mathrm{C}$ for $12 \mathrm{~s}, 60^{\circ} \mathrm{C}$ for $30 \mathrm{~s}, 72^{\circ}$ $\mathrm{C}$ for $40 \mathrm{~s}$, and $1 \mathrm{~s}$ at $81{ }^{\circ} \mathrm{C}$ for plate reading. To ensure the reproducibility of qRT-PCR results, three independent experiments were carried out. The relative expression levels were calculated based on the threshold cycle according to the $2^{-\Delta \Delta \mathrm{CT}}$ method [36].

\section{Analysis of $\mathrm{CdCl}_{2}$ stress tolerance in JrVHAG1 transgenic Arabidopsis plants}

The JrVHAG1 gene was inserted into Arabidopsis plants and three transgenic lines (G2, G6, and G9) with the highest expression level were analyzed [19]. For the seed germination assay, the seeds of WT, G2, G6, and G9 lines were placed on 1/2 Murashige and Skoog (MS) agar medium with 0 (control), or $50 \mu \mathrm{M} \mathrm{CdCl}, 3 \mu \mathrm{M}$ ABA, $50 \mu \mathrm{M} \mathrm{CdCl}_{2}$ plus $3 \mu \mathrm{M} \mathrm{ABA}\left(\mathrm{ABA}+\mathrm{CdCl}_{2}\right)$. Germination rate and average fresh weight of the germinated seedlings were recorded after $12 \mathrm{~d}$ of sowing under standard growth conditions $\left(24{ }^{\circ} \mathrm{C}, 70-75 \%\right.$ relative humidity, and 14/10 h light-dark photoperiod). For comparing seedling growth, 8-day-old seedlings of WT, G2, G6, and G9 were grown on 1/2 MS agar medium and were transferred to 1/2 MS agar medium with 0 (control), $50 \mu \mathrm{M} \mathrm{CdCl}_{2}, 3 \mu \mathrm{M}$ ABA, $50 \mu \mathrm{M} \mathrm{CdCl}_{2}$ plus $3 \mu \mathrm{M}$ ABA $\left(\mathrm{ABA}+\mathrm{CdCl}_{2}\right)$ and grown for another 10 $\mathrm{d}$ after which fresh weight and primary root length were recorded. For analysis of ROS accumulation and variations in physiological performance under abovedescribed treatments, 12-day-old seedlings of WT, G2, G6, and G9 were grown on 1/2 MS, transferred to pots containing a mixture of turf peat and sand $(2: 1 \mathrm{v} / \mathrm{v})$ and grown in a greenhouse for additional 5 -weeks. The seedlings were then treated with 0 (normal watered, control), or $50 \mu \mathrm{M} \mathrm{CdCl}_{2}, 3 \mu \mathrm{M} \mathrm{ABA}, 50 \mu \mathrm{M} \mathrm{CdCl}_{2}$ plus $3 \mu \mathrm{M}$ ABA $\left(\mathrm{ABA}+\mathrm{CdCl}_{2}\right)$ for $6 \mathrm{~d}$, then the aerial parts were collected to determine ROS generation. Histochemical staining for ROS generation in the four lines was performed using DAB and NBT methods [5, 34, 37]. Determination of physiological indices including $\mathrm{H}_{2} \mathrm{O}_{2}$ content, MDA, and proline levels, activity levels of SOD and POD, as well as EL rate was according to previous studies [19, 38]. Every assay was applied at least three times and each replicate contained at least 30 seedlings.

\section{Identification and expression analysis of the JrVHAG1 promoter}

The JrVHAG1 promoter was identified from the walnut genome [39], and amplified by PCR reaction from the $J$. regia DNA. The cis-elements in the JrVHAG1 promoter were analyzed using the PLANTCARE database (http:// bioinformatics.psb.ugent.be/webtools/plantcare/html/) [40]. To understand the expression activity of JrVHAG1 promoter, this promoter was used to replace the $35 S$ promoter 
and cloned into a pCAMBIA1301 vector to drive the expression of GUS gene (Additional file 1: Figure S2). The recombinant construct was transferred into Arabidopsis through Agrobacterium-mediated floral dip method [41]. Four-week-old transgenic seedlings were used to study the expression activity and level through GUS activity determination and staining [5, 42] under normal, $\mathrm{CdCl}_{2}$ and $\mathrm{ABA}$ stress [50 $\mu \mathrm{M}$ $\mathrm{CdCl}_{2}$, or $3 \mu \mathrm{MABA}$, or $50 \mu \mathrm{M} \mathrm{CdCl}_{2}$ plus $3 \mu \mathrm{M}$ $\left.\mathrm{ABA}\left(\mathrm{ABA}+\mathrm{CdCl}_{2}\right)\right]$. Every treatment was replicated three times and each replicate contained at least 30 seedlings.

\section{Identification of the upstream regulator of JrVHAG1}

The core sequence of MYBCORE motif is "CNGTTR", and three MYBCORE elements ("CAGTTG" and "CAGTTA") were found in the JrVHAG1 promoter (Additional file 1: Figure S3). Yeast one-hybrid assay was employed to identify the up-stream transcription factors (TFs) capable of recognizing the MYBCORE motif ("CAGTTG" was used in this study). Three tandem copies of "CAGTTG" were cloned into pHis2 vector (pHis2-MYB) (Additional file 1: Figure S4A). MYB TFs were identified from the $J$. regia transcriptome and cloned into pGADT7-Rec2 vector to generate a cDNA library for use in one-hybrid assays [42].

To confirm the interactions between the motif and positive clones, the MYBCORE "CAGTTG" was mutated to "CCAGGG" and inserted into pHis2 (pHis2-MYB-M). Fragments of the JrVHAG1 promoter including the MYBCORE motif (pHis2-MYB$\mathrm{S}$ ), excluding the MYBCORE motif (pHis2-MYB-M1), and including the mutated MYBCORE motif (pHis2MYB-M2) were all cloned into pHis2, respectively (Additional file 1: Figure S4A). The p53His2 construct was used as a control in the yeast one-hybrid assays [5, 42]. All the primers used are listed in Additional file 1: Table S2.

Furthermore, pHis2-MYB/M/S/M1/M2 were independently fused with a CaMV35S-46 minimal promoter and cloned into pCAMBIA1301 to drive the GUS gene (reportors) expression (Additional file 1: Figure S4C) to confirm the above-described interactions. The ORF of JrMYB2 (screened TF) was cloned into prokII vector such that it is placed under the control of a $35 \mathrm{~S}$ promoter (prokII-JrMYB2) (Additional file 1: Figure S4B), and can act as an effector. Every reportor was transiently co-transformed with the effector in tobacco leaves using Agrobacterium-mediated transformation method, and all co-transformed tobacco leaves were used to measure the GUS activity [42, 43]. Every cotransformation was replicated three times and every replicate contained at least 15 leaves.

\section{Statistical analysis}

All of the data were analyzed using the Statistical Package for Social Science (SPSS) (SPSS, Chicago, Illinois, USA). The differences between the transgenic and WT lines were evaluated using Tukey's multiple comparison test with the significance level set at $p<0.05$, and sample variability is reported as standard deviation (S.D.).

\section{Additional file}

\begin{abstract}
Additional file 1: Table S1. The primers used in GRT-PCR analysis. Table S2. The primers used in yeast one-hybrid assay and PCAMBIA1301 recombinant vector construction. Figure $\mathbf{S} 1$. The abiotic stress tolerance analysis of VHA-B, C, JVHAG 1, Cl and C4 in yeast expression system compared with empty pYES2 (CK) yeast. The six yeast cultures were independently grown in SC-Ura liquid medium containing $2 \%(W / V)$ galactose for $20 \mathrm{~h}$ at $30{ }^{\circ} \mathrm{C}$ to $\mathrm{OD}_{600}=0.4$, then collecting and adjusting the yeast with Sc-Ura including $2 \%$ galactose cultivated to $\mathrm{OD}_{600}=1.6$ for stress analysis. Yeast cell densities $\left(\mathrm{OD}_{600}\right)$ of VHA-B, C, JVH HAG1, $C 1$ and $c 4$ transgenic yeasts and CK were treated with $50 \mu \mathrm{M} \mathrm{ABA}$, or $150 \mu \mathrm{M} \mathrm{CdCl}$, or $150 \mu \mathrm{M} \mathrm{CdCl}_{2}$ plus $50 \mu \mathrm{M} \mathrm{ABA}\left(\mathrm{ABA}+\mathrm{CdCl}_{2}\right)$ for 0 or $24 \mathrm{~h}(0 \mathrm{~h}$ was set as control) were tested. All data are displayed as the mean \pm S.D. of three independent experiments, the significant differences among the six yeasts were compared under the same treatment and indicated by $\mathrm{a}, \mathrm{b}, \mathrm{c}(\boldsymbol{p}<0.05)$, respectively. Figure S2. Mapping the reconstruct of JVHHAG1 promoter that inserted into PCAMBIA1301 vector. Figure S3. The promoter and elements of JrVHAG1 promoter. The MBS means the 'MYBCORE' element. Figure S4. The binding analysis of JrMYB2 to the MYBCORE motif. (A) Diagram of the reportor and effector vectors. Three tandem copies of the MYBCORE were inserted into the pHIS2 vector as the reportor construct. (B)The CDS of JrMYB2 was cloned into pGADT7-Rec2 as the effector construct. The effector and reportor constructs were co-transformed into the yeast strain Y187. (C) Diagram of the reportors and effectors. Triple tandem copies of the W-box were fused with the 355 CaMV-46 minimal promoter and cloned into pCAMBIA1301 for driving the GUS gene as the reportor construct. The CDS of JrMYB2 was cloned into prokll under the control of the $35 \mathrm{~S}$ promoter as the effector constructs. (DOC $1310 \mathrm{~kb}$ )
\end{abstract}

\section{Abbreviations}

3-AT: 3-amino-1, 2, 4-triazole; ABA: Abscisic acid; bHLH: Basic helix-loop-helix; CTAB: Cetyltrimethylammonium ammonium bromide; DAB: 3,3"'-

Diaminobenzidine; EL: Electrolyte leakage; $\mathrm{H}_{2} \mathrm{O}_{2}$ : Hydrogen dioxide; MDA: Malondialdehyde; MS: Murashige and Skoog; NBT: Nitrogen blue tetrazolium; POD: Peroxidase; qRT-PCR: Quantitative Real-Time PCR; ROS: Reactive oxygen species; S.D.: Standard deviation; SD: Synthetic dropout medium; SOD: Superoxide dismutase

\section{Acknowledgements}

Not applicable.

\section{Funding}

The current study was supported by Forestry Science and Technology Project of Hunan Province (XKL201731), Science and Technology Project of Hunan Province (2016TP2007, 2016TP1014), Special Financial Grant from the China Postdoctoral Science Foundation (2017 T100782), Postdoctoral Science Foundation Project of Shaanxi Province (2016BSHEDZZ117). The funding agency was not involved in the design of the study, collection, analysis, and interpretation of data and in writing the manuscript.

Availability of data and materials

All data generated or analysed during this study are included in this published article [and its supplementary information files].

Authors' contributions

ZX wrote the paper and analyzed all the data; YG did the yeast one-hybrid assay and tested the physiological index; YG and WZ did the staining assays 
and all the expression analysis. YZ and GY designed and fund for the current study. All authors read and approved the final manuscript.

\section{Ethics approval and consent to participate}

Not applicable.

\section{Consent for publication}

Not applicable.

\section{Competing interests}

All the authors declare that they have no conflict of interest.

\section{Publisher's Note}

Springer Nature remains neutral with regard to jurisdictional claims in published maps and institutional affiliations.

\begin{abstract}
Author details
${ }^{1}$ Hunan Research Center of Engineering Technology for Utilization of Environmental and Resources Plant, Central South University of Forestry and Technology, 498 Shaoshan South Road, Changsha, Hunan Province 410004, China. ${ }^{2}$ School of Material and Chemical Engineering, Hunan City University, 518 Yingbin Road, Yiyang, Hunan Province 413000, China. ${ }^{3}$ College of Forestry, Hubei University for Nationalities, 39 Xueyuan Road, Enshi, Hubei 445000, China. ${ }^{4}$ Laboratory of Walnut Research Center, College of Forestry, Northwest A \& F University, Yangling, Shaanxi 712100, China.
\end{abstract}

Received: 6 September 2017 Accepted: 11 January 2018 Published online: 22 January 2018

\section{References}

1. Schützendübel A, Polle A. Plant responses to abiotic stresses: heavy metalinduced oxidative stress and protection by mycorrhization. J Exp Bot. 2002; 53(372):1351-65.

2. Foy C, Rt C, White M. The physiology of metal toxicity in plants. Annu Rev Plant Physiol. 1978;29:511-66.

3. Ouelhadj A, Kuschk P, Humbeck K. Heavy metal stress and leaf senescence induce the barley gene HvC2d1 encoding a calcium-dependent novel C2 domain-like protein. New Phytol. 2006;170:261-73.

4. Dartan G, Taspinar F, Toroz I. Assessment of heavy metals in agricultural soils and their source apportionment: a Turkish district survey. Environ Monit Assess. 2015;187:015-4337.

5. Yang G, Wang C, Wang Y, Guo Y, Zhao Y, Yang C, Gao C. Overexpression of ThVHACl and its potential upstream regulator, ThWRKY7, improved plant tolerance of cadmium stress. Sci Rep. 2016;6:18752.

6. Uzinger N, Anton AD, Otvos K, Tamas P, Anton A. Results of the clean-up operation to reduce pollution on flooded agricultural fields after the red mud spill in Hungary. Environ Sci Pollut Res Int. 2015:4:4.

7. Hou J, Liu X, Cui B, Bai J, Wang X. Concentration-dependent alterations in gene expression induced by cadmium in Solanum lycopersicum. Environ Sci Pollut Res Int. 2017:24:10528-36.

8. DalCorso G, Farinati S, Furini A. Regulatory networks of cadmium stress in plants. Plant Signal Behav. 2010;5:663-7.

9. Farinati S, DalCorso G, Varotto S, Furini A. The Brassica juncea BjCdR15, an ortholog of Arabidopsis TGA3, is a regulator of cadmium uptake, transport and accumulation in shoots and confers cadmium tolerance in transgenic plants. New Phytol. 2010;185:964-78.

10. Das N, Bhattacharya S, Bhattacharyya S, Maiti M. Identification of alternatively spliced transcripts of rice phytochelatin synthase 2 gene OsPCS2 involved in mitigation of cadmium and arsenic stresses. Plant Mol Biol. 2017:11:017-0600

11. Dobrikova AG, Yotsova EK, Borner A, Landjeva SP, Apostolova EL. The wheat mutant DELLA-encoding gene (Rht-B1C) affects plant photosynthetic responses to cadmium stress. Plant Physiol Biochem. 2017;114:10-8.

12. Guo Q, Meng L, Humphreys MW, Scullion J, Mur LA. Expression of FIHMA3, a P1B2-ATPase from Festulolium loliaceum, correlates with response to cadmium stress. Plant Physiol Biochem. 2017;112:270-7.

13. Dietz KJ, Tavakoli N, Kluge C, Mimura T, Sharma SS, Harris GC, Chardonnens AN, Golldack D. Significance of the V-type ATPase for the adaptation to stressful growth conditions and its regulation on the molecular and biochemical level. J Exp Bot. 2001;52:1969-80.
14. Dong Q, Wang C, Liu D, Hu D, Fang M, You C, Yao Y, Hao Y. MdVHA-A encodes an apple subunit a of vacuolar $\mathrm{H}^{+}$-ATPase and enhances drought tolerance in transgenic tobacco seedlings. J Plant Physiol. 2013;170:601-9.

15. Wang $L$, He $X$, Zhao $Y$, Shen $Y$, Huang Z. Wheat vacuolar $H^{+}$-ATPase subunit B cloning and its involvement in salt tolerance. Planta. 2011; 234:1-7.

16. Tyagi W, Rajagopal D, Singla-Pareek SL, Reddy MK, Sopory SK. Cloning and regulation of a stress-regulated Pennisetum Glaucum vacuolar ATPase $c$ gene and characterization of its promoter that is expressed in shoot hairs and floral organs. Plant Cell Physiol. 2005;46:1411-22.

17. Hanitzsch M, Schnitzer D, Seidel T, Golldack D, Dietz KJ. Transcript level regulation of the vacuolar $\mathrm{H}(+)$-ATPase subunit isoforms VHA-a, VHA-E and VHA-G in Arabidopsis thaliana. Mol Membr Biol. 2007:24:507-18.

18. Abdallah IB, Tlili N, Martinez-Force E, Rubio AG, Perez-Camino MC, Albouchi A, Boukhchina S. Content of carotenoids, tocopherols, sterols, triterpenic and aliphatic alcohols, and volatile compounds in six walnuts (Juglans regia L.) varieties. Food Chem. 2015;15(173):972-8.

19. Xu Z, Zhao Y, Ge Y, Peng J, Dong M, Yang G. Characterization of a vacuolar $H$ ${ }^{+}$-ATPase $\mathrm{G}$ subunit gene from Juglans regia (JVHHAG1) involved in mannitolinduced osmotic stress tolerance. Plant Cell Rep. 2016;36(3):407-18.

20. Li P, Song A, Gao C, Jiang J, Chen S, Fang W, Zhang F, Chen F. The overexpression of a chrysanthemum WRKY transcription factor enhances aphid resistance. Plant Physiol Biochem. 2015;95:26-34.

21. Kabala K, Janicka-Russak M, Reda M, Migocka M. Transcriptional regulation of the V-ATPase subunit $c$ and V-PPase isoforms in Cucumis Sativus under heavy metal stress. Physiol Plant. 2014;150:32-45.

22. Yang G, Zhang W, Liu Z, Yi-Maer A-Y, Zhai M, Xu Z. Both JrWRKY2 and JrWRKY7 of Juglans Regia mediate responses to abiotic stresses and abscisic acid through formation of homodimers and interaction. Plant Biol (Stuttgart, Germany). 2017;19(2):268-78.

23. Zhou A, Bu Y, Takano T, Zhang X, Liu S. Conserved V-ATPase c subunit plays a role in plant growth by influencing $\mathrm{V}$-ATPase-dependent endosomal trafficking. Plant Biotechnol J. 2015;28:12381.

24. Pompeu GB, Vilhena MB, Gratao PL, Carvalho RF, Rossi ML, Martinelli AP, Azevedo RA. Abscisic acid-deficient sit tomato mutant responses to cadmium-induced stress. Protoplasma. 2017:254:771-83.

25. Deng B, Yang K, Zhang Y, Li Z. Can heavy metal pollution defend seed germination against heat stress? Effect of heavy metals $(\mathrm{cu}(2+), \mathrm{Cd}(2+)$ and $\mathrm{hg}(2+))$ on maize seed germination under high temperature. Environ Pollut. 2016;216:46-52

26. Khan N, Bano A. Role of plant growth promoting rhizobacteria and Ag-nano particle in the bioremediation of heavy metals and maize growth under municipal wastewater irrigation. Int J Phytoremediation. 2016;18:211-21.

27. Yao P, Li C, Zhao X, Li M, Zhao H, Guo J, Cai Y, Chen H, Wu Q. Overexpression of a tartary buckwheat gene, FtbHLH3, enhances drought/oxidative stress tolerance in transgenic Arabidopsis. Front Plant Sci. 2017;25(8):625.

28. Lin T, Yang W, Lu W, Wang Y, Qi X. Transcription factors PVERF15 and PVMTF-1 form a cadmium stress transcriptional pathway. Plant Physiol. 2017;173:1565-73.

29. Nakano Y, Yamaguchi M, Endo H, Rejab NA, Ohtani M. NAC-MYB-based transcriptional regulation of secondary cell wall biosynthesis in land plants. Front Plant Sci. 2015;6:288

30. Zamioudis C, Hanson J, Pieterse CM. Beta-Glucosidase BGLU42 is a MYB72dependent key regulator of rhizobacteria-induced systemic resistance and modulates iron deficiency responses in Arabidopsis roots. New Phytol. 2014; 204:368-79.

31. Chen $Y$, Wu $X$, Ling $H$, Yang W. Transgenic expression of DwMYB2 impairs iron transport from root to shoot in Arabidopsis thaliana. Cell Res. 2006;16:830-40

32. Shukla PS, Agarwal P, Gupta K, Agarwal PK. Molecular characterization of an MYB transcription factor from a succulent halophyte involved in stress tolerance. AoB Plants. 2015;17:plv054

33. Yang G, Xu Z, Peng S, Sun Y, Jia C, Zhai M. In planta characterization of a tau class glutathione S-transferase gene from Juglans Regia (JrGSTTau1) involved in chilling tolerance. Plant Cell Rep. 2016:35:681-92.

34. Yang G, Wang Y, Xia D, Gao C, Wang C, Yang C. Overexpression of a GST gene (ThGSTZ1) from Tamarix hispida improves drought and salinity tolerance by enhancing the ability to scavenge reactive oxygen species. Plant Cell Tissue Organ Cult (PCTOC). 2014:117:99-112.

35. Xu F, Deng G, Cheng S, Zhang W, Huang X, Li L, Cheng H, Rong X, Li J. Molecular cloning, characterization and expression of the phenylalanine ammonia-lyase gene from Juglans regia. Molecules. 2012;17(7):7810-23. 
36. Livak KJ, Schmittgen TD. Analysis of relative gene expression data using realtime quantitative PCR and the $2^{-\Delta \Delta C T}$ method. Methods. 2001;25:402-8.

37. Zhang X, Wang L, Meng H, Wen H, Fan Y, Zhao J. Maize ABP9 enhances tolerance to multiple stresses in transgenic Arabidopsis by modulating ABA signaling and cellular levels of reactive oxygen species. Plant Mol Biol. 2011; 75:365-78.

38. Yang $G, Y u L$, Wang $Y$, Wang $C$, Gao C. The translation initiation factor $1 A$ (ThelF1A) from Tamarix hispida is regulated by a Dof transcription factor and increased abiotic stress tolerance. Front Plant Sci. 2017:8:513.

39. Martinez-Garcia PJ, Crepeau MW, Puiu D, Gonzalez-Ibeas D, Whalen J, Stevens KA, Paul R, Butterfield TS, Britton MT, Reagan RL, Chakraborty S, Walawage SL, Vasquez-Gross HA, Cardeno C, Famula RA, Pratt K, Kuruganti S, Aradhya MK, Leslie CA, Dandekar AM, Salzberg SL, Wegrzyn JL, Langley CH, Neale DB. The walnut (Juglans regia) genome sequence reveals diversity in genes coding for the biosynthesis of non-structural polyphenols. Plant $J$. 2016;87:507-32.

40. Lescot M, Dehais P, Thiis G, Marchal K, Moreau Y, Van de Peer Y, Rouze P, Rombauts S. PlantCARE, a database of plant cis-acting regulatory elements and a portal to tools for in silico analysis of promoter sequences. Nucleic Acids Res. 2002;30:325-7.

41. Clough SJ, Bent AF. Floral dip: a simplified method forAgrobacteriummediated transformation of Arabidopsis thaliana. Plant J. 1998;16:735-43.

42. Zheng L, Liu G, Meng X, Liu Y, Ji X, Li Y, Nie X, Wang Y. A WRKY gene from Tamarix hispida, ThWRKY4, mediates abiotic stress responses by modulating reactive oxygen species and expression of stress-responsive genes. Plant Mol Biol. 2013;82:303-20.

43. Jefferson R. The GUS reporter gene system. Nature. 1989;342:837.

\section{Submit your next manuscript to BioMed Central and we will help you at every step:}

- We accept pre-submission inquiries

- Our selector tool helps you to find the most relevant journal

- We provide round the clock customer support

- Convenient online submission

- Thorough peer review

- Inclusion in PubMed and all major indexing services

- Maximum visibility for your research

Submit your manuscript at www.biomedcentral.com/submit

C) Biomed Central 